\title{
MIXED METHODS FOR THE APPROXIMATION OF LIQUID CRYSTAL FLOWS *
}

\author{
Chun LiU ${ }^{1}$ and Noel J. Walkington ${ }^{2}$
}

\begin{abstract}
The numerical solution of the flow of a liquid crystal governed by a particular instance of the Ericksen-Leslie equations is considered. Convergence results for this system rely crucially upon energy estimates which involve $H^{2}(\Omega)$ norms of the director field. We show how a mixed method may be used to eliminate the need for Hermite finite elements and establish convergence of the method.
\end{abstract}

Mathematics Subject Classification. 65M60, 76A15.

Received: December 11, 2000. Revised: January 7, 2002.

\section{INTRODUCTION}

We consider the problem of approximating the following instance of the Ericksen-Leslie equations which model the motion of liquid crystal flows [20,29,30]:

$$
\begin{gathered}
u_{t}+(u \cdot \nabla) u+\nabla p-\operatorname{div}\left(\nu D(u)-\lambda(\nabla d)^{T} \nabla d\right)=0, \\
\nabla \cdot u=0 \\
d_{t}+(u . \nabla) d-\gamma(\Delta d-f(d))=0
\end{gathered}
$$

with initial and boundary conditions

$$
\left.u\right|_{t=0}=u_{0},\left.\quad d\right|_{t=0}=d_{0},\left.\quad u\right|_{\partial \Omega}=0,\left.\quad d\right|_{\partial \Omega}=d_{0}
$$

Here $u$ represents the velocity of the liquid crystal flow, $p$ the pressure, and $d$ represents the orientation of the liquid crystal molecules: $u, d: \Omega \times \Re^{+} \rightarrow \Re^{n}, p: \Omega \times \Re^{+} \rightarrow \Re$ and $\Omega \subset \Re^{n}$. In the above, $D(u)=$ $(1 / 2)\left(\nabla u+(\nabla u)^{T}\right)$ is the stretching tensor,

$$
(\nabla d)_{i j}=d_{i, j}=\frac{\partial d_{i}}{\partial x_{j}}, \quad \text { so that } \quad\left((\nabla d)^{T} \nabla d\right)_{i j}=\sum_{k=1}^{n} \frac{\partial d_{k}}{\partial x_{i}} \frac{\partial d_{k}}{\partial x_{j}}
$$

Keywords and phrases. Liquid Crystals, Mixed Finite Element Approximation, Convergence.

* Supported in part by National Science Foundation Grant No. DMS-9972040.w and No. DMS-9973285. This work was also supported by the NSF through the Center for Nonlinear Analysis.

1 Department of Mathematics, Pennsylvania State, University, University Park, PA 18601, USA.

2 Department of Mathematics, Carnegie Mellon University, Pittsburgh, PA 15213, USA. 
The penalty function is typically chosen to be $f(d)=\left(1 / \epsilon^{2}\right)\left(|d|^{2}-1\right) d$ which approximates the constraint $|d|=1$ and is the gradient of the scalar valued function $F(d)=\left(1 / 4 \epsilon^{2}\right)\left(|d|^{2}-1\right)^{2}$. For the analysis of the numerical schemes below it is convenient to assume that $F$ is truncated to have quadratic growth at infinity. Equations (1.1) describe the balance of mass and momentum of a liquid crystal occupying the region $\Omega$. The first of these equations is the Navier Stokes equation describing the balance of linear momentum, and the divergence free condition on the velocity represents the balance of mass for an incompressible fluid. The third equation determines the evolution of the director field and would normally contain a Lagrange multiplier dual to the constraint $|d|=1$; however, for reasons discussed below, we chose to approximate this constraint using penalization.

While these equations are a simplification of the full Ericksen-Leslie system, it is important to note that they contain all of the essential mathematical structure. In particular, the existence theory for these equations developed by Lin and Liu [33] has been extended to the complete Ericksen-Leslie system [34], and more recently by Liu [36] to equations modeling smectic liquid crystals which have additional structure. In this situation a detailed understanding of the numerical approximation of equations (1.1) is of importance.

The crucial observation underpinning the results of Lin and Liu $[33,34]$ is that solutions of the above system are dissipative in the sense that

$$
\frac{\mathrm{d} E}{\mathrm{~d} t}=-\left(\nu\|D(u)\|_{L^{2}(\Omega)}^{2}+\lambda \gamma\|\Delta d-f(d)\|_{L^{2}(\Omega)}^{2}\right),
$$

where

$$
E=(1 / 2)\|u\|_{L^{2}(\Omega)}^{2}+(\lambda / 2)\|\nabla d\|_{L^{2}(\Omega)}^{2}+\lambda \int_{\Omega} F(d) .
$$

In order for this estimate to hold it is essential that the director field have two square integrable derivatives, i.e. $d \in H^{2}(\Omega)$. To establish similar estimates for numerical approximations of equations (1.1) in two dimensions the authors used Hermite finite elements to construct subspaces of $H^{2}(\Omega)$ [37]. While Hermite finite elements are quite accurate since they are constructed from high degree polynomials, they do suffer several disadvantages. Firstly, their basis function are complicated to construct and typically values of the function, its first derivatives, and the mixed second derivatives are required at the grid points of a finite element mesh and this complicates the computation of initial and boundary data. When derivatives appear as degrees of freedom, the basis functions all scale differently and specification of boundary values is non-trivial, or at least tedious, and these problems compound when going from two to three dimensions.

In this paper we circumvent the use of Hermite finite elements by constructing mixed approximations where derivatives of the director field are approximated "independently" of the director. Defining (the matrix) $W=$ $\nabla d$, the mixed approximation can be viewed as an approximation of equations (1.1) written in the form:

$$
\begin{gathered}
u_{t}+(u \cdot \nabla) u+\nabla p-\nu \operatorname{div} D(u)+\lambda(\nabla d)^{T} \operatorname{div}(W)=0, \\
\nabla \cdot u=0, \quad \nabla d=W, \\
W_{t}+\nabla((\nabla d) u-\gamma(\operatorname{div} W-f(d)))=0,
\end{gathered}
$$

with boundary and initial conditions

$$
\left.u\right|_{t=0}=u_{0},\left.\quad W\right|_{t=0}=\nabla d_{0},\left.\quad u\right|_{\partial \Omega}=0,\left.\quad d\right|_{\partial \Omega}=d_{0} .
$$

Notice that $\Delta d=\operatorname{div}(W)=\left(W_{i j, j}\right)$ and that we have not replaced $(\nabla d)$ with $W$ in the terms coupling the velocity and director equations. This is important in the analysis below where care is required in order to guarantee that certain terms in the corresponding weak problemare integrable. (In the language of [8], the weak 
problems we construct are only well posed by virtue of elliptic regularity theory.) In equations (1.3) we wrote

$$
\operatorname{div}\left((\nabla d)^{T} \nabla d\right)=(\nabla d)^{T} \Delta d+\nabla\left(|\nabla d|^{2}\right) / 2=(\nabla d)^{T} \operatorname{div}(W)+\nabla\left(|\nabla d|^{2}\right) / 2
$$

and absorbed the gradient into the pressure $\left(p \sim p+|\nabla d|^{2} / 2\right)$.

The study of liquid crystals has a long history, and we refer the reader to [9] for a broad overview from an engineering perspective. Initially static configurations were studied $[21,25,38]$, and these models were extended by Ericksen and Leslie $[20,29]$ to the more general dynamical situation. These models all characterize the orientation of a liquid crystal molecule by a unit vector field; however, more general descriptions have been proposed to explain phenomena not compatible with this description. The most common generalization is to use traceless tensors [16] which can, for example, model "degree 1/2" singularities that can not be represented by vector fields. Paralleling the analysis, most of the numerical simulations of liquid crystals to date have focused on the stationary case $[1,12,15,35]$ and are based upon the variational theory for the static case $[24,25]$. The equations describing the motion of liquid crystals have much in common with the harmonic mapping problem and the equations describing the density of super conducting electrons, each of which involve constrained vector fields and hence involve singularities [4-7, 17-19,26,41].

\subsection{Role of penalization}

The fundamental work of Lin and Liu [33] provides the mathematical framework for our analysis. In [33] it is shown that equations (1.1), which approximate the constraint $|d|=1$ using penalization, enjoy existence and uniqueness of solutions for all time, and regularity is also established. The energy estimate (1.2) provides bounds upon the solution that are independent of the penalty parameter $\epsilon$; however, estimates comparing solutions having different initial data do depend upon $\epsilon$. This explains why the error estimates for numerical approximations of these equations will also depend upon $\epsilon$. Another place where constants depending upon the penalty terms, and hence $\epsilon$, appear is in the discrete energy estimate. In the continuous situation one has the identity

$$
\int_{\Omega} F(d(\tau))-F(d(0))=\int_{0}^{\tau} \frac{\mathrm{d}}{\mathrm{d} t} \int_{\Omega} F(d(t)) \mathrm{d} t=\int_{0}^{\tau} \int_{\Omega} f(d) \cdot d_{t} \mathrm{~d} t,
$$

however, for the discrete problem if $d^{1} \sim d(\tau)$ and $d^{0} \sim d(0)$ then

$$
\int_{\Omega} F\left(d^{1}\right)-F\left(d^{0}\right) \leq \leq \int_{\Omega} f\left(u^{1}\right) \cdot\left(u^{1}-u^{0}\right),
$$

due to the lack of convexity of $F$.

While the energy estimate (1.2) provides bounds for $d$ in the space $L^{\infty}\left[0, T ; L^{2}(\Omega)\right] \cap L^{2}\left[0, T ; H^{2}(\Omega)\right]$, this is not enough to identify limits of all the nonlinear terms appearing in the equations. Lin and Liu also showed that $d_{t}$ is bounded in $L^{4 / 3}\left[0, T ; L^{2}(\Omega)\right]$, independently of $\epsilon$, which, when combined with "wedge product" techniques developed for harmonic maps $[10,42]$, provides sufficient compactness for passage to the limit. In particular, the limiting equation for $d$ is

$$
d_{t}+(u . \nabla) d-\gamma(\Delta d-\theta d)=0, \quad|d|=1 \text { (a.e.) },
$$

where $\theta$ is a Lagrange multiplier and is known to equal $|\nabla d|^{2}$ in this particular situation [33,34]. Compactness arguments do not provide rates of convergence of the penalized solution to the limiting solution, and for this reason it is not possible to estimate how close a solution of the equations with finite $\epsilon$ are to a solution of the limit equation. There is however a deeper reason. The liquid crystal configurations of most interest are those containing singularities and in two dimensions these do not have finite energy, so in this situation the limit equation only holds in the sense of measures; in particular, the weak forms that we exploit below to construct numerical schemes would not be well posed for the limit equation. This phenomenon has been studied for the 
Ginzburg-Landau equation,

$$
d_{t}-\Delta d=\left(1 / \epsilon^{2}\right)\left(1-|d|^{2}\right) d
$$

In two dimensions the disparate scaling between the finite "interaction" energy and the infinite "core" energy makes it possible to introduce a "renormalized" energy, and to derive equations describing the motion of the singularities for this equation [27,31,32]. Currently similar results are not yet available for the equations describing the motion of liquid crystals, and there are very few results for the three dimensional problem where the interaction and core energies are both finite.

While it is clear that the penalized equations (1.1) allow singularities to be approximated in all dimensions with continuous vector fields having finite energy, one other property they enjoy, unlike the limit equations, is uniqueness of solution. Lin and Liu [33,34] showed that smooth solutions of the limit equations will be unique; however, the question of uniqueness in the presence of singularities is still an open question ${ }^{1}$. A more serious problem for schemes to approximate the limit equations is that the stationary problem is known to have multiple solutions [28]. Parabolic equations are almost universally approximated by solving a sequence of (stationary) elliptic problems, and non-convexity of the constraint $|d|=1$ and the lack of uniqueness are known to plague numerical schemes for the approximation of static liquid crystal configurations $[1,2,12,13]$.

\subsection{Notation}

While our results are applicable to arbitrary dimension, we are particularly interested in the three dimensional situation $(n=3)$, and, for ease of exposition, below we assume that this is the case. Throughout we will assume that $\Omega \subset \Re^{3}$ is a bounded Lipschitz domain with boundary $\partial \Omega$ sufficiently smooth for $H^{2}$ regularity for the Laplacian to hold. $u, d:[0, T] \times \Omega \rightarrow \Re^{3}$ will denote the velocity and director fields of a liquid crystal and $p:[0, T] \times \Omega \rightarrow \Re$ the pressure. The mixed finite element method employed introduces the director gradient $W=\nabla d$ with components $W_{\alpha \beta}=\partial d_{\alpha} / \partial x_{\beta}$. When constructing Galerkin methods the variables $v, q, e$, and $Z$ will be the dual variables (test functions) corresponding to the velocity, pressure, director, and director gradient respectively. Writing $u \in L^{2}(\Omega)$ for a vector valued quantity, such as the velocity $u$, will indicate that each component lies in the indicated space, and standard notation is adopted for the classical Lebesgue and Sobolev spaces. Similarly, write $W \in H^{1}(\Omega ; \operatorname{div})=\left\{w: \Omega \rightarrow \Re^{3} \mid w \in L^{2}(\Omega)\right.$, div $\left.w \in L^{2}(\Omega)\right\}$, to indicate that $W_{\alpha}, \in H^{1}(\Omega ; \operatorname{div})$ for each $\alpha$.

The variables $u, p, d$ and $W$ will always refer to the solution of the continuous problem and a superscript, such as $u^{n}$, will refer to the approximation of $u\left(t^{n}\right)=u(n \tau)$ obtained using a time stepping scheme. The fully discrete approximation of $u$ at this time will denoted by or $u_{h}^{n}$. In order to keep the notation manageable, inside a proof we may write $u$ for $u\left(t^{n}\right), u_{h}$ for $u_{h}^{n}$ etc. if a line of argument is independent of the time step under consideration. Below we will also introduce projections $(\tilde{u}, \tilde{p}, \tilde{d}, \tilde{W})$ of the solution $(u, p, d, W)$ onto the finite element spaces, and again $\tilde{u}^{n}=\tilde{u}\left(t^{n}\right)$ etc.

The symmetric part of the velocity gradient is denoted by $D(u)=(1 / 2)\left(\nabla u+(\nabla u)^{T}\right)$, and Korn's inequality, $\|u\|_{H_{0}^{1}(\Omega)} \leq C\|D(u)\|_{L^{2}(\Omega)}, u \in H_{0}^{1}(\Omega)$, will be used to obtain coercivity of bilinear forms. The inequality $\|e\|_{L^{4}(\Omega)} \leq C\|e\|_{H_{0}^{1}(\Omega)}^{1 / 4}\|e\|_{H^{2}(\Omega)}^{3 / 4}, e \in H_{0}^{1}(\Omega) \cap H^{2}(\Omega)$, will be used below to bound the nonlinear terms. We finally mention that frequent use will be made of the elementary inequality $a b \leq(\eta / 2) a^{2}+(1 / 2 \eta) b^{2}$.

\subsection{Outline}

In the next section we introduce weak forms corresponding to the equations (1.3) that constitute the mixed method under consideration. The weak form was carefully constructed so that Galerkin approximations would inherit energy estimates, and the resulting bounds are then established. In Section 3 we establish convergence of the Galerkin finite element approximations of the solutions of (1.3) which is our main result. A numerical

\footnotetext{
${ }^{1}$ In two dimensions uniqueness can be established for singular solutions having infinite energy.
} 
example is presented in Section 4 demonstrating the use of the mixed method to compute the motion of singularities.

\section{ENERGy ESTIMATES AND WEAK PROBLEMS}

\subsection{Weak forms}

We construct a (mixed) weak formulation of the equations (1.1) which will be approximated by our numerical scheme. Temporal derivatives are approximated using the implicit Euler approximation, and we will seek solutions of the resulting sequence of elliptic equations in the space

$$
\mathcal{V}=\mathcal{U} \times \mathcal{P} \times \mathcal{W} \times \mathcal{D}=H_{0}^{1}(\Omega) \times L^{2}(\Omega) / \Re \times H^{1}(\Omega ; \operatorname{div}) \times H_{0}^{1}(\Omega) .
$$

Specifically, given the solution at time $t^{n}=n \tau$, let $\left(u^{n+1}, p^{n+1}, W^{n+1}, d^{n+1}-d_{0}\right) \in \mathcal{V}$ satisfy

$$
\begin{gathered}
\int_{\Omega} u \cdot v+\tau\left((1 / 2)((u \cdot \nabla) u \cdot v-(u \cdot \nabla) v \cdot u)-p \nabla \cdot v+\nu D(u) \cdot D(v)+\lambda \operatorname{div}(W)^{T}(\nabla d) v\right)=\int_{\Omega} u^{n} \cdot v, \\
\int_{\Omega} \nabla \cdot u q=0, \\
\int_{\Omega} W \cdot Z+\tau(-(\nabla d) u+\gamma(\operatorname{div}(W)-f(d))) \cdot \operatorname{div}(Z)=\int_{\Omega} W^{n} \cdot Z, \\
\int_{\Omega} \nabla d \cdot \nabla e-\operatorname{div}(W) \cdot e=0 .
\end{gathered}
$$

for all $(v, q, Z, e) \in \mathcal{V}$. The identity $\int_{\Omega}(u . \nabla) u . v=-\int_{\Omega}(u . \nabla) v \cdot u$ when $\operatorname{div}(u)=0$ has been used to guarantee that the convective term remains anti-symmetric for velocities $u \in H_{0}^{1}(\Omega)$ that may not be divergence free.

At first glance this weak form may not appear to be well defined since the terms of the form $(\nabla d) u$. $\operatorname{div}(Z)$ coupling the momentum and director gradient equation are not a priori integrable. However, note that if we do have a solution of the weak problem, then the last equation implies that $\Delta d=\operatorname{div}(W) \in L^{2}(\Omega)$. Regularity theory for the Laplacian then implies $\|d\|_{H^{2}(\Omega)} \leq C\left(\|\operatorname{div}(W)\|_{L^{2}(\Omega)}+\left\|d_{0}\right\|_{H^{2}(\Omega)}\right)$ and since $H^{2}(\Omega) \hookrightarrow W^{1,4}(\Omega)$ and $u \in H_{0}^{1}(\Omega) \hookrightarrow L^{4}(\Omega)$ it follows that $(\nabla d) u$. $\operatorname{div}(Z)$ is integrable. The bounds established below show that $\|\operatorname{div}(W)\|_{L^{2}(\Omega)}$ can be bounded by the datum $d_{0}$, and then standard techniques can be used to establish existence of a unique solution of this weak problem. While this line of argument suffices for the continuous case, it will need to be modified when constructing Galerkin approximations since the Galerkin subspaces will not be contained in $H^{2}(\Omega)$. An alternative argument will be used to obtain the $W_{0}^{1,4}(\Omega)$ bounds required for the convergence analysis.

Approximate solutions of (1.1) are found by computing solutions of the weak problem (2.1) restricted to finite dimensional subspaces $\mathcal{V}_{h}=\mathcal{U}_{h} \times \mathcal{P}_{h} \times \mathcal{W}_{h} \times \mathcal{D}_{h} \subset \mathcal{V}$ constructed using finite element spaces. Since the spaces are finite dimensional, the terms coupling the momentum and director equation are integrable so the discrete problem is well defined. Existence of the discrete solutions may be established using Brower's fixed point Theorem and the energy estimates established next. Since such arguments are standard we do not repeat them here.

\subsection{Bounds}

Assuming, that each of the terms in the weak form are integrable, bounds on the solution follow upon substituting $(v, q, Z, e)=\left(u, p, \lambda W, d-d_{0}\right)$ :

$$
\frac{1}{2}\left(\|u\|_{L^{2}(\Omega)}^{2}+\lambda\|W\|_{L^{2}(\Omega)}^{2}\right)+\tau\left(\nu\|D(u)\|_{L^{2}(\Omega)}^{2}+\lambda \gamma\|\operatorname{div}(W)\|_{L^{2}(\Omega)}^{2}\right) \leq \frac{1}{2}\left\|u^{n}\right\|_{L^{2}(\Omega)}^{2}+\tau \lambda \gamma \int_{\Omega} f(d) . \operatorname{div}(W),
$$


and $\left\|\nabla\left(d-d_{0}\right)\right\|_{L^{2}(\Omega)} \leq\|W\|_{L^{2}(\Omega)}+\left\|\nabla d_{0}\right\|_{L^{2}(\Omega)}$. If we assume that $f=D F$ is smooth and globally Lipschitz then $|f(d)| \leq C(F)\left(\left|d_{0}\right|+\left|d-d_{0}\right|\right)$, and an application of the Poincaré inequality enables the penalty term to be bounded by

$$
\begin{aligned}
\int_{\Omega} f(d) \cdot \operatorname{div}(W) & \leq C(F)\left(\left\|d_{0}\right\|_{L^{2}(\Omega)}+\|W\|_{L^{2}(\Omega)}\right)\|\operatorname{div}(W)\|_{L^{2}(\Omega)} \\
& \leq C\left(d_{0}, F\right)^{2}+C(F)\|W\|_{L^{2}(\Omega)}^{2}+(1 / 2)\|\operatorname{div}(W)\|_{L^{2}(\Omega)}^{2} .
\end{aligned}
$$

The discrete Gronwall inequality then shows that

$$
\begin{aligned}
\left(\left\|u^{n}\right\|_{L^{2}(\Omega)}^{2}+\left\|W^{n}\right\|_{L^{2}(\Omega)}^{2}\right)+\sum_{m=1}^{N} \tau\left(\nu\left\|D\left(u^{m}\right)\right\|_{L^{2}(\Omega)}^{2}+\right. & \left.\lambda \gamma\left\|\operatorname{div}\left(W^{m}\right)\right\|_{L^{2}(\Omega)}^{2}\right) \\
& \leq C(F)\left(\left\|u^{0}\right\|_{L^{2}(\Omega)}^{2}+\left\|W^{0}\right\|_{L^{2}(\Omega)}^{2}\right)+C\left(d_{0}, F\right)
\end{aligned}
$$

where $N=T / \tau$ and $0 \leq n \leq N$. Unlike the bound (1.2), this estimate is applicable to both the continuous problem and to the Galerkin approximations of the weak problem. In the above we have indicated where the constants may depend upon $F$, and in particular the penalty parameter $\epsilon$ appearing in its definition.

\section{Convergence of the Galerkin approximations}

\subsection{Notation and discrete spaces}

In order to make the structure of the proofs more concise we introduce some notation to abstract out the essential features.

\section{Notation 3.1.}

- If $\mathbf{u}=(u, W)$ and $\mathbf{v}=(v, Z)$ define the bilinear form on $\mathcal{U} \times \mathcal{W}=H_{0}^{1}(\Omega) \times H^{1}(\Omega$; div $)$ by

$$
A(\mathbf{u}, \mathbf{v})=\int_{\Omega} \nu D(u) \cdot D(v)+\lambda \gamma \operatorname{div} W \cdot \operatorname{div} Z,
$$

and the bilinear form on $\mathcal{P} \times \mathcal{U}$ by

$$
B(p, \mathbf{v})=B(p, v)=\int_{\Omega} p \operatorname{div}(v) .
$$

- Define the (nonlinear) operator $G: \mathcal{U} \times \mathcal{W} \times \mathcal{D} \rightarrow(\mathcal{U} \times \mathcal{W})^{\prime}$ by

$$
\begin{aligned}
\langle G(\mathbf{u}, d), \mathbf{v}\rangle= & \left.\int_{\Omega}(1 / 2)[(u . \nabla) u \cdot v-(u \cdot \nabla) v \cdot u)\right] \\
& +\lambda\left((\operatorname{div} W)^{T}(\nabla d) v-(\nabla d) u \cdot \operatorname{div} Z\right)-\lambda \gamma f(d) . \operatorname{div} Z .
\end{aligned}
$$

- The inner product (.,.) on $\mathcal{U} \times \mathcal{W}$ is given by

$$
(\mathbf{u}, \mathbf{v})=\int_{\Omega} u \cdot v+\lambda W \cdot Z,
$$

and the norm induced by the bilinear form $A(.,$.$) is denoted by \|$.$\| . The dual norm of \|$.$\| will be denoted$ by $\|.\|_{*}$, i.e. if $g \in(\mathcal{U} \times \mathcal{W})^{\prime}$ then $\|g\|_{*}=\sup _{\mathbf{v}} \mid\langle g, \mathbf{v}\rangle /\|\mathbf{v}\|$. 
Solutions of the continuous problem will satisfy

$$
\left(\mathbf{u}_{t}(t), \mathbf{v}\right)+A(\mathbf{u}(t), \mathbf{v})+B(p(t), \mathbf{v})+\langle G(\mathbf{u}(t), d), \mathbf{v}\rangle=0, \quad B(q, \mathbf{u}(t))=0,
$$

and

$$
\int_{\Omega} \nabla d(t) \cdot \nabla e+\operatorname{div} W(t) \cdot e=0
$$

for all $(v, q, Z, e) \in \mathcal{V}$, and the discrete solutions satisfy $\left(u_{h}, p_{h}, W_{h}, d_{h}-d_{0}\right) \in \mathcal{V}_{h}$

$$
\frac{1}{\tau}\left(\mathbf{u}_{h}-\mathbf{u}_{h}^{n}, \mathbf{v}\right)+A\left(\mathbf{u}_{h}, \mathbf{v}\right)+B\left(p_{h}, \mathbf{v}\right)+\left\langle G\left(\mathbf{u}_{h}, d_{h}\right), \mathbf{v}\right)=0, \quad B\left(q, \mathbf{u}_{h}\right)=0,
$$

and

$$
\int_{\Omega} \nabla d_{h} \cdot \nabla e+\operatorname{div} W_{h} \cdot e=0
$$

for all $(v, q, Z, e) \in \mathcal{V}_{h}$.

\subsection{Discrete spaces}

The proof of convergence will use some standard properties of the finite element spaces used to construct the discrete space $\mathcal{V}_{h}$. As mentioned above, the use of a mixed method allows the use of standard Lagrangian based finite element schemes, and we introduce notation for the natural projections onto these spaces and recall some of their essential properties. Specific examples of such spaces are detailed in Section 4 where some numerical examples are presented.

We will assume that the discrete spaces for the velocity and pressure satisfy the Babuska-Brezzi condition $[3,8,22]$ :

$$
\sup _{u_{h} \in \mathcal{U}_{h}} \frac{\int_{\Omega}\left(\nabla \cdot u_{h}\right) p_{h}}{\left\|u_{h}\right\|_{H_{0}^{1}(\Omega)}} \geq c\left\|p_{h}\right\|_{L^{2}(\Omega) / \Re} \quad p_{h} \in \mathcal{P}_{h}
$$

and will assume that the director gradient $W$ is approximated using one of the Raviart Thomas, or more generally the $B D F M$, family of subspaces of $H^{1}(\Omega ;$ div $)$ [8].

Finally we introduce notation for the projections used ubiquitously for the analysis of finite element schemes.

\section{Notation 3.2.}

- (1) Given $u \in \mathcal{U}$ then the elliptic projection onto the discretely divergence free subspace satisfies $\tilde{u} \in \mathcal{U}_{h}$

$$
\int_{\Omega} \nu D(\tilde{u}) \cdot D\left(v_{h}\right)+\tilde{p}\left(\nabla \cdot v_{h}\right)=\int_{\Omega} \nu D(u) \cdot D\left(v_{h}\right), \quad \int_{\Omega} q_{h}(\nabla . \tilde{u})=0,
$$

for some $\tilde{p} \in \mathcal{P}_{h}$ and all $\left(v_{h}, q_{h}\right) \in \mathcal{U}_{h} \times \mathcal{P}_{h}$.

(2) If $W \in \mathcal{W}$ then the BDFM family of spaces admit a projection $\tilde{W} \in \mathcal{W}_{h}$ satisfying [8, Proposition 3.9, page 129]

$$
\int_{\Omega} \operatorname{div} \tilde{W} \cdot \operatorname{div} Z_{h}=\int_{\Omega} \operatorname{div} W \cdot \operatorname{div} Z_{h}, \quad Z_{h} \in \mathcal{W}_{h}
$$

The results in [8] pertain specifically to divergences of vector valued functions $w \in H^{1}(\operatorname{div} ; \Omega)$; the above is obtained by applying these results to each row of $W$.

The projection $\Pi_{h}: \mathcal{U} \times \mathcal{W} \rightarrow \mathcal{U}_{h} \times \mathcal{W}_{h}$ is defined by $(\tilde{u}, \tilde{W})=\Pi_{h}(u, W)$ where $\tilde{u}$ and $\tilde{W}$ are the projections given by (1) and (2). 
- (1) $P_{h}: \mathcal{D} \rightarrow \mathcal{D}_{h}$ denotes the elliptic projection; that is, $\hat{d}=P_{h} d \in \mathcal{D}_{h}$ satisfies

$$
\int_{\Omega} \nabla \hat{d} \cdot \nabla e_{h}=\int_{\Omega} \nabla d \cdot \nabla e_{h}, \quad e_{h} \in \mathcal{D}_{h}
$$

(2) We will make frequent use of the approximation $\tilde{d}$ of $d$ defined by $\tilde{d}=P_{h} \delta$ where $\delta-d_{0} \in H_{0}^{1}(\Omega)$ satisfies $-\Delta \delta=\operatorname{div} \tilde{W}$ (with $\tilde{W}$ being the projection of $W$ defined above). Notice that $\nabla\left(P_{h} d\right)$ is the $L^{2}(\Omega)$ projection of $W=\nabla d$ onto the gradients of $\mathcal{D}_{h}$, and $\nabla \tilde{d}$ is the projection of $\tilde{W}$ onto the same space.

Recall that if $\nabla \cdot u=0$, then the elliptic projection $\tilde{u}$ of $u$ onto the discretely divergence free space satisfies

$$
\|u-\tilde{u}\|_{H_{0}^{1}(\Omega)} \leq C \inf _{v_{h} \in \mathcal{U}_{h}}\left\|u-v_{h}\right\|_{H_{0}^{1}(\Omega)},
$$

whenever the spaces $\mathcal{U}_{h}$ and $\mathcal{P}_{h}$ satisfy the Babuska-Brezzi condition $[22,45]$. Similarly, the projection of $\mathcal{W}=H^{1}(\Omega$; div) onto the $B D F M$ family of spaces exhibit optimal rates of convergence [8].

\subsection{Constraint equation}

Recall that the terms coupling the momentum and director equation in the weak form (2.1) were, for the continuous spaces, integrable only by virtue of the regularity of $\nabla d$. While this isn't a problem for the (finite dimensional) discrete problem, it is still necessary to bound higher norms of the coupling terms in order to establish error estimates. In this section such bounds are established using properties of the constraint equation. These arguments do not establish $L^{4}(\Omega)$ bounds on $W$ and this is why the term $(\nabla d) u$ in the director equation is not written as $W u$. The key idea is to use the non-trivial fact that the elliptic projection $P_{h}$ is continuous on all of the $W_{0}^{1, p}(\Omega)$ spaces $[14,39,40]$, i.e. $\left\|P_{h} d\right\|_{W_{0}^{1, p}(\Omega)} \leq C(p, \Omega)\|d\|_{W_{0}^{1, p}(\Omega)}$ (the constant independent of $h$ for regular meshes). This property is frequently used to estimate nonlinear terms having polynomial growth.

\section{$H^{1}(\Omega)$ Estimates}

Let $\tilde{d}$ and $\tilde{W}$ be the projections of $d$ and $W$ onto the discrete spaces $\mathcal{D}_{h}$ and $\mathcal{W}_{h}$ defined in Notation 3.2. Then

$$
\int_{\Omega} \nabla\left(\tilde{d}-d_{h}\right) \cdot \nabla e_{h}=\int_{\Omega} \nabla\left(\hat{d}-d_{h}\right) \cdot \nabla e_{h}=\int_{\Omega} \operatorname{div}\left(\tilde{W}-W_{h}\right) \cdot e_{h}, \quad e_{h} \in \mathcal{D}_{h} .
$$

Putting $e_{h}=\tilde{d}-d_{h}$ gives

$$
\left\|\tilde{d}-d_{h}\right\|_{H_{0}^{1}(\Omega)}^{2}=\int_{\Omega} \operatorname{div}\left(\tilde{W}-W_{h}\right) \cdot\left(\tilde{d}-d_{h}\right)
$$

and

$$
\left\|\tilde{d}-d_{h}\right\|_{H_{0}^{1}(\Omega)} \leq\left\|\tilde{W}-W_{h}\right\|_{L^{2}(\Omega)},
$$

where integration by parts was used to obtain the last line. An identical calculation shows that $\left\|P_{h} d-\tilde{d}\right\|_{H_{0}^{1}(\Omega)} \leq$ $\|W-\tilde{W}\|_{L^{2}(\Omega)}$.

\section{$L^{2}(\Omega)$ Estimates}

The Aubin-Nitche trick [11] is used to estimate the $L^{2}(\Omega)$ error. Let $e \in H_{0}^{1}(\Omega)$ satisfy $-\Delta e=\tilde{d}-d_{h}$, and recall that since $\Omega$ is a Lipschitz domain that $\|e\|_{H^{2}(\Omega)} \leq C\left\|\tilde{d}-d_{h}\right\|_{L^{2}(\Omega)}$. Letting $e_{h}=P_{h} e$ be the elliptic projection of $e$, then $\left\|e_{h}\right\|_{H_{0}^{1}(\Omega)} \leq\left\|\tilde{d}-d_{h}\right\|_{L^{2}(\Omega)}$, and standard finite element theory establishes that 
$\left\|e-e_{h}\right\|_{H_{0}^{1}(\Omega)} \leq C h\|e\|_{H^{2}(\Omega)} \leq C h\left\|\tilde{d}-d_{h}\right\|_{L^{2}(\Omega)}$. Then

$$
\begin{aligned}
\left\|\tilde{d}-d_{h}\right\|_{L^{2}(\Omega)}^{2} & =\int_{\Omega} \nabla\left(\tilde{d}-d_{h}\right) \cdot \nabla e \\
& =\int_{\Omega} \nabla\left(\tilde{d}-d_{h}\right) \cdot \nabla\left(e-e_{h}\right)-\operatorname{div}\left(\tilde{W}-W_{h}\right) \cdot e_{h}, \\
\left\|\tilde{d}-d_{h}\right\|_{L^{2}(\Omega)} & \leq C h\left\|\tilde{d}-d_{h}\right\|_{H_{0}^{1}(\Omega)}+\left\|\tilde{W}-W_{h}\right\|_{L^{2}(\Omega)} \\
& \leq(1+C h)\left\|\tilde{W}-W_{h}\right\|_{L^{2}(\Omega)} .
\end{aligned}
$$

Similarly we obtain $\left\|P_{h} d-\tilde{d}\right\|_{L^{2}(\Omega)} \leq\|W-\tilde{W}\|_{L^{2}(\Omega)}$ and classical finite element theory [11] establishes that $\left\|d-P_{h} d\right\|_{L^{2}(\Omega)} \leq C h \inf _{e_{h} \in \mathcal{D}_{h}}\left\|d-e_{h}\right\|_{H_{0}^{1}(\Omega)}$ so that

$$
\|d-\tilde{d}\|_{L^{2}(\Omega)} \leq C\left(h \inf _{e_{h} \in \mathcal{D}_{h}}\left\|d-e_{h}\right\|_{H_{0}^{1}(\Omega)}+\|W-\tilde{W}\|_{L^{2}(\Omega)}\right) .
$$

$W_{0}^{1,4}(\Omega)$ Estimates. To estimate $\left\|\tilde{d}-d_{h}\right\|_{W_{0}^{1,4}(\Omega)}$ define $\delta \in H_{0}^{1}(\Omega)$ by $-\Delta \delta=\operatorname{div}\left(\tilde{W}-W_{h}\right)$. Integration by parts shows that $\|\delta\|_{H_{0}^{1}(\Omega)} \leq\left\|\tilde{W}-W_{h}\right\|_{L^{2}(\Omega)}$, and regularity theory gives $\|\delta\|_{H^{2}(\Omega)} \leq C\left\|\operatorname{div}\left(\tilde{W}-W_{h}\right)\right\|_{L^{2}(\Omega)}$. Next, observe that $\tilde{d}-d_{h}=P_{h} \delta$, so that

$$
\begin{aligned}
\left\|\tilde{d}-d_{h}\right\|_{W_{0}^{1,4}(\Omega)} & \leq C\|\delta\|_{W_{0}^{1,4}(\Omega)} \\
& \leq C\|\delta\|_{H_{0}^{1}(\Omega)}^{1 / 4}\|\delta\|_{H^{2}(\Omega)}^{3 / 4} \\
& \leq C\left\|\tilde{W}-W_{h}\right\|_{L^{2}(\Omega)}^{1 / 4}\left\|\operatorname{div}\left(\tilde{W}-W_{h}\right)\right\|_{L^{2}(\Omega)}^{3 / 4},
\end{aligned}
$$

The estimate $\|e\|_{L^{4}(\Omega)} \leq C\|e\|_{H_{0}^{1}(\Omega)}^{1 / 4}\|e\|_{H^{2}(\Omega)}^{3 / 4}$ for $e \in H_{0}^{1}(\Omega) \cap H^{2}(\Omega)$ was used in the second step above. We may substitute $\left(d_{h}, W_{h}\right)$ for $\left(P_{h} d, W\right)$ in the argument to obtain

$$
\begin{aligned}
\|d-\tilde{d}\|_{W_{0}^{1,4}(\Omega)} & \leq\left\|d-P_{h} d\right\|_{W_{0}^{1,4}(\Omega)}+\left\|P_{h} d-\tilde{d}\right\|_{W_{0}^{1,4}(\Omega)} \\
& \leq\left\|d-P_{h} d\right\|_{W_{0}^{1,4}(\Omega)}+C\|W-\tilde{W}\|_{L^{2}(\Omega)}^{1 / 4}\|\operatorname{div}(W-\tilde{W})\|_{L^{2}(\Omega)}^{3 / 4} .
\end{aligned}
$$

\subsection{Estimating the error}

To obtain an expression for the error between the solutions of the continuous and discrete weak problems, observe that by restricting the test functions in equation (3.1) to lie in $\mathcal{V}_{h}$ one obtains

In the above

$$
\begin{gathered}
\left(\mathbf{u}_{t}(t), \mathbf{v}\right)+A(\tilde{\mathbf{u}}(t), \mathbf{v})+B(p(t), \mathbf{v})+\langle G(\mathbf{u}(t), d), \mathbf{v}\rangle=0, \quad \mathbf{v} \in \tilde{\mathcal{U}}_{h} \\
B(q, \tilde{u}(t))=0 \quad q \in \mathcal{P}_{h}
\end{gathered}
$$

$$
\tilde{\mathcal{U}}_{h}=\left\{u_{h} \in \mathcal{U}_{h} \mid B\left(q_{h}, u_{h}\right)=0, \forall q_{h} \in \mathcal{P}_{h}\right\}
$$

is the discretely divergence free space and $\tilde{\mathbf{u}}=(\tilde{u}, \tilde{W})=\Pi_{h} \mathbf{u}$ is the projection of the exact solution $(u, W)$ onto $\tilde{\mathcal{U}}_{h} \times \mathcal{W}_{h}$ given in Notation 3.2. Evaluating this equation at time $t=t^{n+1}$ and subtracting equation (3.2) gives

$$
\frac{1}{\tau}\left(\mathbf{e}^{n+1}-\mathbf{e}^{n}, \mathbf{v}\right)+A\left(\mathbf{e}^{n+1}, \mathbf{v}\right)+\left\langle G\left(\mathbf{u}\left(t^{n+1}\right), d\left(t^{n+1}\right)\right)-G\left(\mathbf{u}_{h}^{n+1}, d_{h}^{n+1}\right), \mathbf{v}\right\rangle=-\left(\varepsilon^{n+1}, \mathbf{v}\right)-B\left(p-q_{h}, \mathbf{v}\right),
$$


for all $\mathbf{v} \in \tilde{\mathcal{U}}_{h} \times \mathcal{W}$ and $q_{h} \in \mathcal{P}_{h}$. In this equation $\mathbf{e}^{n+1}=\tilde{\mathbf{u}}\left(t^{n+1}\right)-\mathbf{u}_{h}^{n+1}$ and $\varepsilon^{n+1}$ is the consistency error of the linear part of the equation:

$$
\begin{aligned}
\varepsilon^{n+1} & =\mathbf{u}_{t}\left(t^{n+1}\right)-\frac{\tilde{\mathbf{u}}\left(t^{n+1}\right)-\tilde{\mathbf{u}}\left(t^{n}\right)}{\tau} \\
& =\frac{1}{\tau} \int_{t^{n}}^{t^{n+1}}\left(I d-\Pi_{h}\right) \mathbf{u}_{t}(s) \mathrm{d} s+\int_{t^{n}}^{t^{n+1}} \frac{s-t^{n}}{\tau} \mathbf{u}_{t t}(s) \mathrm{d} s .
\end{aligned}
$$

Here $I d$ is the identity operator and the solution of the continuous problem is assumed to have sufficient regularity for each of the terms to make sense. Putting $\mathbf{v}=\mathbf{e}_{h}^{n+1}$ into the equation for the error gives, after an application of the Cauchy Schwarz inequality,

$$
\begin{aligned}
\frac{1}{2 \tau}\left|\mathbf{e}^{n+1}\right|^{2}+\left\|\mathbf{e}^{n+1}\right\|^{2} \leq \frac{1}{2 \tau}\left|\mathbf{e}^{n}\right|^{2}+\left\|p-q_{h}\right\|_{L^{2}(\Omega)}\left\|\mathbf{e}^{n+1}\right\|+\left\|\varepsilon^{n+1}\right\|_{*}\left\|\mathbf{e}^{n+1}\right\| & \\
& +\left|\left\langle G\left(\mathbf{u}\left(t^{n+1}\right), d\left(t^{n+1}\right)\right)-G\left(\mathbf{u}_{h}^{n+1}, d_{h}^{n+1}\right), \mathbf{e}^{n+1}\right\rangle\right|
\end{aligned}
$$

so that

$$
\frac{1}{2 \tau}\left|\mathbf{e}^{n+1}\right|^{2}+\frac{1}{2}\left\|\mathbf{e}^{n+1}\right\|^{2} \leq \frac{1}{2 \tau}\left|\mathbf{e}^{n}\right|^{2}+\left\|p-q_{h}\right\|_{L^{2}(\Omega)}^{2}+\left\|\varepsilon^{n+1}\right\|_{*}^{2}+\left|\left\langle G\left(\mathbf{u}\left(t^{n+1}\right), d\left(t^{n+1}\right)\right)-G\left(\mathbf{u}_{h}^{n+1}, d_{h}^{n+1}\right), \mathbf{e}^{n+1}\right\rangle\right| .
$$

The following Lemma bounds the last (nonlinear) term in the above equation and will then be used to establish error estimates.

Lemma 3.3. If the solution of (1.3) satisfies

$$
u \in C\left[0, T ; H_{0}^{1}(\Omega)\right], \quad W \in C\left[0, T ; H^{1}(\Omega ; \operatorname{div})\right], \quad d-d_{0} \in C\left[0, T ; W_{0}^{1,4}(\Omega)\right],
$$

then the nonlinear terms in equation (3.7) satisfy

$$
\begin{aligned}
& \left|\left\langle G\left(\mathbf{u}\left(t^{n+1}\right), d\left(t^{n+1}\right)\right)-G\left(\mathbf{u}_{h}^{n+1}, d_{h}^{n+1}\right), \mathbf{e}^{n+1}\right\rangle\right| \leq(1 / 4)\left\|\mathbf{e}^{n+1}\right\|^{2}+ \\
& \quad C\left(\left|\mathbf{e}^{n+1}\right|^{2}+\left\|d\left(t^{n+1}\right)-\tilde{d}\left(t^{n+1}\right)\right\|_{W_{0}^{1,4}(\Omega)}^{2}+\left\|\mathbf{u}\left(t^{n+1}\right)-\tilde{\mathbf{u}}\left(t^{n+1}\right)\right\|^{2}\right) .
\end{aligned}
$$

where $\tilde{\mathbf{u}}=(\tilde{u}, \tilde{W})$ and $\tilde{d}$ are the projections defined in Notation 3.2.

Proof. In order to reduce the notation, write $\mathbf{e}^{n+1}=\mathbf{e},\left(\mathbf{u}\left(t^{n+1}\right), d\left(t^{n+1}\right)\right)=(\mathbf{u}, d)$ and $\left(\mathbf{u}_{h}^{n+1}, d_{h}^{n+1}\right)=\left(\mathbf{u}_{h}, d_{h}\right)$ for the remainder of the proof. Next, let $\tilde{\mathbf{u}}=\Pi_{h} \mathbf{u}$ and $\tilde{d}$ be the projections introduced in Notation (3.2). We write

$$
\left\langle G(\mathbf{u}, d)-G\left(\mathbf{u}_{h}, d_{h}\right), \mathbf{e}\right\rangle=\langle G(\mathbf{u}, d)-G(\tilde{\mathbf{u}}, \tilde{d}), \mathbf{e}\rangle-\left\langle G(\tilde{\mathbf{u}}, \tilde{d})-G\left(\mathbf{u}_{h}, d_{h}\right), \mathbf{e}\right\rangle,
$$

and will estimate each term separately. The above expressions involve many terms, and a detailed examination of each of them would be somewhat repetitive and uninteresting. Accordingly we will suppress the some of the details and emphasize how the structure of the nonlinearity allows the proof to go through and, in particular, how the terms can be arranged so that a coefficient no bigger than $1 / 4$ multiplies the term $\|\mathbf{e}\|^{2}$. Throughout the proof we will make frequent use of the inequality $a b \leq(a / \eta)^{p} / p+(\eta b)^{p^{\prime}} / p^{\prime}$ for arbitrary $a, b, \eta>0$ and exponents $1 \leq p \leq \infty, 1 / p+1 / p^{\prime}=1$. The constant $\eta$ will appear as the coefficient of $\|\mathbf{e}\|^{2}$ six times (two for each of the convective term, the coupling term, and the penalty term), so it may be chosen to be sufficiently small to get a grand total of no more than $1 / 4$.

Consider first the terms coupling the momentum and director equation in the expression $\langle G(\tilde{\mathbf{u}}, \tilde{d})$ $\left.-G\left(\mathbf{u}_{h}, d_{h}\right), \mathbf{e}\right\rangle$. Observe that $\mathbf{e}=\tilde{\mathbf{u}}-\mathbf{u}_{h} \equiv\left(e_{u}, E_{W}\right)$, and in this situation the same cancellation property 
used to obtain the bounds (2.2) holds.

$$
\begin{aligned}
& \int_{\Omega}\left((\operatorname{div} \tilde{W})^{T}(\nabla \tilde{d}) e_{u}-(\nabla \tilde{d}) \tilde{u} \cdot \operatorname{div} E_{W}-\left(\operatorname{div} W_{h}\right)^{T}\left(\nabla d_{h}\right) e_{u}+\left(\nabla d_{h}\right) u_{h} \cdot \operatorname{div} E_{W}\right) \\
& =\int_{\Omega}\left((\operatorname{div} \tilde{W})^{T}\left(\nabla\left(\tilde{d}-d_{h}\right)\right) e_{u}-\left(\nabla\left(\tilde{d}-d_{h}\right)\right) \tilde{u} \cdot \operatorname{div} E_{W}\right) \\
& \leq\left\|\nabla\left(\tilde{d}-d_{h}\right)\right\|_{L^{4}(\Omega)}\left(\|\operatorname{div} \tilde{W}\|_{L^{2}(\Omega)}\left\|e_{u}\right\|_{L^{4}(\Omega)}+\left\|\operatorname{div} E_{W}\right\|_{L^{2}(\Omega)}\|\tilde{u}\|_{L^{4}(\Omega)}\right) \\
& \leq C\left\|E_{W}\right\|_{L^{2}(\Omega)}^{1 / 4}\left\|\operatorname{div} E_{W}\right\|_{L^{2}(\Omega)}^{3 / 4} \\
& \quad \times\left(\|\operatorname{div} \tilde{W}\|_{L^{2}(\Omega)}\left\|e_{u}\right\|_{L^{4}(\Omega)}+\left\|\operatorname{div} E_{W}\right\|_{L^{2}(\Omega)}\|\tilde{u}\|_{L^{4}(\Omega)}\right) \\
& \leq C(\eta)\left(\left\|E_{W}\right\|_{L^{2}(\Omega)}^{2}+\left\|e_{u}\right\|_{L^{2}(\Omega)}^{2}+\right)+\eta\left(\left\|\nabla e_{u}\right\|_{L^{2}(\Omega)}^{2}+\left\|\operatorname{div} E_{W}\right\|_{L^{2}(\Omega)}^{2}\right) \\
& \leq C(\eta)|\mathbf{e}|^{2}+\eta\|\mathbf{e}\|^{2} .
\end{aligned}
$$

The cancellation can be observed in the second line of the above, and the $W_{0}^{1,4}(\Omega)$ estimates (3.4) established in the previous section were used in an essential fashion in the fourth line. We have tacitly made use of Korn's inequality $[11,23]$ which shows that the norms $\left\|\nabla e_{u}\right\|_{L^{2}(\Omega)}$ and $\left\|D\left(e_{u}\right)\right\|_{L^{2}(\Omega)}$ are equivalent. The convective terms in the momentum equation are accommodated in an identical fashion.

We consider next the corresponding terms in the expression $\langle G(\mathbf{u}, d)-G(\tilde{\mathbf{u}}, \tilde{d})$, e $\rangle$. The lack of cancellation is compensated for by the assumed regularity of the exact solution.

$$
\begin{aligned}
\int_{\Omega} & \left((\operatorname{div} W)^{T}(\nabla d) e_{u}-(\nabla d) u \cdot \operatorname{div} E_{W}-(\operatorname{div} \tilde{W})^{T}(\nabla \tilde{d}) e_{u}+(\nabla \tilde{d}) \tilde{u} \cdot \operatorname{div} E_{W}\right) \\
= & \int_{\Omega}\left((\operatorname{div}(W-\tilde{W}))^{T}(\nabla d) e_{u}+(\operatorname{div} \tilde{W})^{T}(\nabla(d-\tilde{d})) e_{u}\right. \\
& \left.-(\nabla d)(u-\tilde{u}) \cdot \operatorname{div} E_{W}-(\nabla(d-\tilde{d})) \tilde{u} \cdot \operatorname{div} E_{W}\right) \\
\leq & \left(\|\nabla(d-\tilde{d})\|_{L^{4}(\Omega)}\|\operatorname{div} \tilde{W}\|_{L^{2}(\Omega)}+\|\operatorname{div}(W-\tilde{W})\|_{L^{2}(\Omega)}\|\nabla d\|_{L^{4}(\Omega)}\right)\left\|e_{u}\right\|_{L^{4}(\Omega)} \\
& +\left(\|\nabla(d-\tilde{d})\|_{L^{4}(\Omega)}\|\tilde{u}\|_{L^{4}(\Omega)}+\|\nabla d\|_{L^{4}(\Omega)}\|u-\tilde{u}\|_{L^{4}(\Omega)}\right)\left\|\operatorname{div} E_{W}\right\|_{L^{2}(\Omega)} \\
\leq & C(\eta)\left(\|\nabla(d-\tilde{d})\|_{L^{4}(\Omega)}^{2}+\|\operatorname{div}(W-\tilde{W})\|_{L^{2}(\Omega)}^{2}+\|u-\tilde{u}\|_{L^{4}(\Omega)}^{2}\right) \\
& +\left\|e_{u}\right\|_{L^{2}(\Omega)}^{2}+\eta\left(\left\|\nabla e_{u}\right\|_{L^{2}(\Omega)}^{2}+\left\|\operatorname{div} E_{W}\right\|_{L^{2}(\Omega)}^{2}\right) \\
\leq & C(\eta)\left(\|\nabla(d-\tilde{d})\|_{L^{4}(\Omega)}^{2}+\|\mathbf{u}-\tilde{\mathbf{u}}\|^{2}\right)+\left\|e_{u}\right\|_{L^{2}(\Omega)}^{2}+\eta\|\mathbf{e}\|^{2} .
\end{aligned}
$$

The Sobolev embedding theorem (in three dimensions), $\left\|e_{u}\right\|_{L^{4}(\Omega)} \leq\left\|e_{u}\right\|_{L^{2}(\Omega)}^{1 / 4}\left\|\nabla e_{u}\right\|_{L^{2}(\Omega)}^{3 / 4}$ and Korn's inequality were used in the above, and, again, the convective terms in the momentum equation are handled similarly.

Finally, the bound on the penalty term follows from the Lipschitz hypothesis on $f=D F$.

$$
\begin{aligned}
\int_{\Omega}\left(f(\tilde{d})-f\left(d_{h}\right)\right) \cdot \operatorname{div} E_{W} & \leq\|D f\|_{L^{\infty}(\Omega)}\left\|\tilde{d}-d_{h}\right\|_{L^{2}(\Omega)}\left\|\operatorname{div} E_{W}\right\|_{L^{2}(\Omega)} \\
& \leq C(\eta)\left\|\tilde{d}-d_{h}\right\|_{L^{2}(\Omega)}^{2}+\eta\left\|\operatorname{div} E_{W}\right\|_{L^{2}(\Omega)}^{2} \\
& \leq C(\eta)\left\|\tilde{W}-W_{h}\right\|_{L^{2}(\Omega)}^{2}+\eta\left\|\operatorname{div} E_{W}\right\|_{L^{2}(\Omega)}^{2} \\
& \leq C(\eta)\left\|E_{W}\right\|_{L^{2}(\Omega)}^{2}+\eta\left\|\operatorname{div} E_{W}\right\|_{L^{2}(\Omega)}^{2} .
\end{aligned}
$$


To estimate the corresponding term involving $f(d)-f(\tilde{d})$ we would simply stop above computation after second line of the above with $(d, W)$ being substituted for $\left(d_{h}, W_{h}\right)$.

Given this technical lemma we can now state the main theorem.

Theorem 3.4. Let $\left\{\left(u_{h}^{n}, p_{h}^{n}, W_{h}^{n}, d_{h}^{n}\right)\right\}_{n=0}^{N} \subset \mathcal{V}_{h}, N=T / \tau$ be finite element approximation of equations (3.2) modeling the flow of the liquid crystal crystals computed using the weak problem (2.1). Let the finite element meshes $\left\{\mathcal{T}_{h}\right\}_{h>0}$ used to construct subspaces $\mathcal{V}_{h} \subset \mathcal{V}$ form a regular family, and let $\Omega$ be a Lipschitz domain sufficiently smooth for $H^{2}$-regularity of the Laplacian to hold. Assume that the spaces $\mathcal{V}_{h}=\mathcal{U}_{h} \times \mathcal{P}_{h} \times \mathcal{W}_{h} \times \mathcal{D}_{h}$ are constructed using elements containing polynomials of degree $k$ for $\mathcal{U}_{h}$ and $\mathcal{D}_{h}$, polynomials of degree $k-1$ for $\mathcal{P}_{h}$, and that $\mathcal{W}_{h}$ is constructed from the BDFM family of finite elements and that the pair $\left(\mathcal{U}_{h}, \mathcal{P}_{h}\right)$ satisfy the Babuska Brezzi condition.

If the solution of the system of (3.1) satisfies $p \in C\left[0, T ; H^{k}(\Omega)\right]$,

$$
\begin{aligned}
& u \in H^{2}\left[0, T ; H^{-1}(\Omega)\right] \cap H^{1}\left[0, T ; H^{k}(\Omega)\right] \cap C\left[0, T ; H^{k+1}(\Omega)\right], \\
& d \in H^{2}\left[0, T ; L^{2}(\Omega)\right] \cap H^{1}\left[0, T ; H^{k+1}(\Omega)\right] \cap C\left[0, T ; W^{k+1,4}(\Omega)\right],
\end{aligned}
$$

and the initial interpolant of the initial data satisfies

$$
\left\|u(0)-u_{h}^{0}\right\|_{L^{2}(\Omega)}+\left\|W(0)-W_{h}^{0}\right\|_{L^{2}(\Omega)} \leq C h^{k},
$$

then

$$
\begin{aligned}
\max _{1 \leq n \leq N}\left(\left\|u\left(t^{n}\right)-u_{h}^{n}\right\|_{L^{2}(\Omega)}+\right. & \left.\left\|W\left(t^{n}\right)-W_{h}^{n}\right\|_{L^{2}(\Omega)}\right) \\
& +\left(\sum_{m=1}^{N} \tau\left(\left\|\nabla\left(u\left(t^{n}\right)-u_{h}^{n}\right)^{2}\right\|_{L^{2}(\Omega)}+\left\|\operatorname{div}\left(W\left(t^{n}\right)-W_{h}^{n}\right)\right\|_{L^{2}(\Omega)}^{2}\right)\right)^{1 / 2} \leq C\left(\tau+h^{k}\right),
\end{aligned}
$$

and

$$
\max _{1 \leq n \leq N}\left\|d\left(t^{n}\right)-d_{h}^{n}\right\|_{H^{1}(\Omega)} \leq C h^{k}
$$

Proof. By the discrete Gronwall inequality it suffices to verify that the right hand side of equation (3.7) can be bounded by an expression of the form

$$
\left(1 /(2 \tau)+C_{1}^{n+1}\right)\left|\mathbf{e}^{n}\right|^{2}+C_{2}^{n+1}\left(\tau+h^{k}\right)^{2}+(1 / 4)\left\|\mathbf{e}^{n+1}\right\|^{2} .
$$

where $C_{1}^{n}$ and $C_{2}^{n}$ are sumable in the sense that $\sum_{m=0}^{N} \tau C_{\alpha}^{m}<\infty$. We consider each term on appearing on the right of (3.7) in turn.

Error in the pressure: The regularity hypothesis on $p$ and interpolation theory [11] show that

$$
\inf _{q_{h} \in \mathcal{P}_{h}}\left\|p\left(t^{n+1}\right)-q_{h}\right\|_{L^{2}(\Omega)} \leq C h^{k}
$$


Consistency error of the linear terms: Recalling equation (3.6) and the notation $\mathbf{u}=(u, W)$, the consistency error can be bounded by

$$
\begin{aligned}
\left\|\varepsilon^{n+1}\right\|_{*}^{2} \leq & \frac{1}{\tau} \int_{t^{n}}^{t^{n+1}}\left\|\left(I-\Pi_{h}\right) \mathbf{u}_{t}(t)\right\|_{L^{2}(\Omega)}^{2} \mathrm{~d} t+\tau \int_{t^{n}}^{t^{n+1}}\left\|\mathbf{u}_{t t}(t)\right\|_{*}^{2} \mathrm{~d} t \\
\leq & \frac{1}{\tau} \int_{t^{n}}^{t^{n+1}}\left(\left\|u_{t}\right\|_{H^{k}(\Omega)}^{2}+\left\|W_{t}\right\|_{H^{k}(\Omega)}^{2}\right) \mathrm{d} t\left(C h^{k}\right)^{2} \\
& +\tau \int_{t^{n}}^{t^{n+1}}\left(\left\|u_{t t}\right\|_{H^{-1}(\Omega)}^{2}+\left\|W_{t t}\right\|_{H^{1}(\Omega ; \operatorname{div})^{\prime}}\right) \mathrm{d} t .
\end{aligned}
$$

Observe that the hypothesis on $d$ do bound the (somewhat obscure) norm $\left\|W_{t t}\right\|_{H^{1}(\Omega ; \text { div) }}$, since for $Z \in$ $H^{1}(\Omega ; \operatorname{div})$

$$
\left(W_{t t}, Z\right)=\left(\nabla d_{t t}, Z\right)=\left(d_{t t}, \operatorname{div} Z\right) \leq\left\|d_{t t}\right\|_{L^{2}(\Omega)}\|Z\|_{H^{1}(\Omega ; \operatorname{div})} .
$$

Recall that the Dirichlet boundary condition on $d$ is independent of time so $d_{t t}$ vanishes on $\partial \Omega$ eliminating the boundary term that would otherwise arise upon integration by parts.

Penalty term: Upon appealing to Lemma (3.3) we deduce that it is sufficient to estimate

$$
\left\|d\left(t^{n+1}\right)-\tilde{d}\left(t^{n+1}\right)\right\|_{W_{0}^{1,4}(\Omega)}^{2}+\left\|\mathbf{u}\left(t^{n+1}\right)-\tilde{\mathbf{u}}\left(t^{n+1}\right)\right\|^{2},
$$

(recall that $\mathbf{u}=(u, W)$ ). Equation (3.5) shows that estimating this expression reduces to interpolation estimates. Standard interpolation theory [11], interpolation theory in the BDFM spaces [8], and interpolation theory in the presence of the divergence constraint [22], and the regularity assumed on $d, u$ and $W$, show that both of the terms appearing in this expression are bounded by the square of $C h^{k}$.

We finish this section with a few comments about the proof. First, note that the estimate on $\|\varepsilon\|_{*}$ was obtained by estimating the first term in (3.6) in $L^{2}(\Omega)$. While this is optimal under the current assumptions, if $\Omega$ is smooth enough for $H^{3}$ regularity then similar estimates hold under less regularity on the exact solution. Secondly, by exploiting super convergence results, it may be possible to approximate the director $d$ with polynomials of degree one less than indicated in the theorem. This is suggested by the loss in precision when passing from equation (3.4) to equation (3.5).

\section{NUMERICAL EXAMPLE}

In this section we present an example illustrating how the mixed method can be used to compute solutions containing singularities and, in particular, their motion. Three dimensional computations of liquid crystal flows are expensive since they involve the resolution of two vector fields in addition to the scalar pressure. Moreover, the use of either Hermite elements or a mixed method for the director field adds to the computational demands. We use a Newton scheme to solve the nonlinear system at each time step. The computations presented here used a banded Gauss elimination routine to solve the associated systems of linear equations. However, this required a lot of memory and was slow, so we subsequently utilized an iterative linear algebra package realizing a significant improvement in performance.

\subsection{Finite element spaces}

We consider domains $\Omega=[0,1]^{2}$ or $[0,1]^{3}$ and their uniform subdivision into finite elements. It is then natural to consider tensor product elements. Approximating spaces for the velocity and pressure are constructed using the ubiquitous $Q_{2} \times Q_{1}$ element. The velocity space is constructed from tensor products of quadratic polynomials, and the pressure space from tensor products of linear polynomials; both functions are globally continuous. This pair of spaces is known to satisfy the Babuska Brezzi condition $[43,44]$. The biquadratic $Q_{2}$ element was used to 


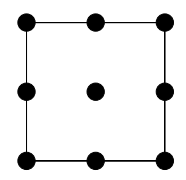

Velocity

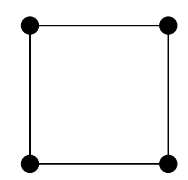

Pressure

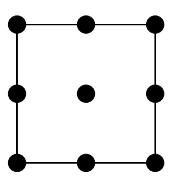

Director

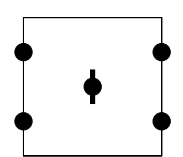

x-Gradients

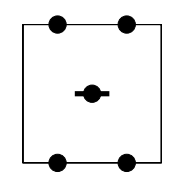

y-Gradients

Figure 1. Two dimensional elements (dot corresponds to a function value, dot with bar indicates average value in indicated direction).

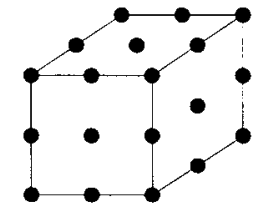

Velocity

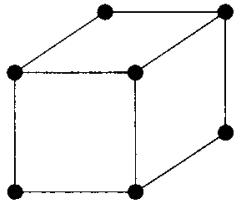

Pressure

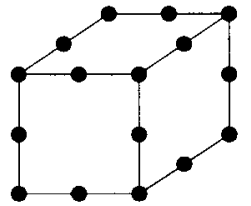

Director

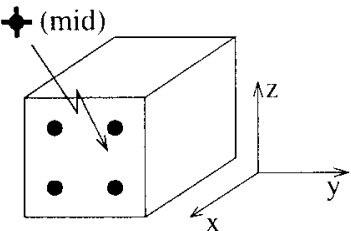

$\mathrm{x}$-Gradients

Figure 2. Three dimensional elements (dot corresponds to a function value, dot with bars indicates average value in indicated plane).

construct approximating spaces for the director $d$ in two dimensions, and the serendipity $Q_{2}^{\prime}$ element is used to construct approximating spaces for the director in three dimensions for efficiency reasons. The director gradient $W$ was approximated using the $B D F M_{1}$ family of finite elements in two dimensions and their natural extension in three dimension. This space of functions is constructed from tensor products of linear polynomials which are then augmented to give a quadratic rate of convergence for both $W$ and $\operatorname{div} W$ in $L^{2}(\Omega)$. These elements are shown in Figures 1 and 2; notice that the $B D F M_{1}$ elements represent each component of a vector differently and that the functions are only continuous in the direction of that component. In Figures 1 and 2 the solid dots indicate that the degree of freedom is the function value, and circles with bars indicate that the average of the function in the direction of the dash is the degree of freedom ${ }^{2}$. This choice of elements gives a second order rate of convergence which respect to the element size $h$ for the norms indicated in Theorem 3.4.

\section{Annihilation of singularities}

We illustrate how singularities of opposite sign in the director field will move together an annihilate each other. To construct vector fields we considered singularities of the form $d(x)=\tilde{d}(x) / \sqrt{|\tilde{d}(x)|^{2}+\varepsilon^{2}}$ where

$$
\tilde{d}(x)=w(x) A^{-}(x+c)+(1-w(x)) A^{+}(x-c) .
$$

Here $w(x, y, z)=1 /(1+\exp (5 x))$ approximates a partition of unity and the matrices $A^{ \pm}$have diagonals $(1, \pm 1)$ in two dimensions and $(1, \pm 1,1)$ in three dimensions. The vector $c$ is set to $c=(1 / 2,0)$ or $(1 / 2,0,0)$ in two and three dimensions respectively. This gives singularities on the axis at $x \simeq \pm 0.5635394250$. Figure 3a plots the initial director field on a $5 \times 5$ grid for the two dimensional calculation; a section through the plane $z=0$ for a $5 \times 5 \times 5$ three dimensional mesh looks very similar. Figure $3 \mathrm{~b}$ shows the final director field for the two dimensional problem at time $t=1$, and it is clear that the singularities have been annihilated to produce a smooth degree zero vector field. For all of the computations presented here the parameters $\lambda, \nu$ and $\gamma$ are

\footnotetext{
${ }^{2}$ Note that it is not possible to approximate the average by the value at the mid point without suffering a loss of accuracy. However, approximating the average by the two (four) point Gauss rule in two (three) dimensions is permissible.
} 

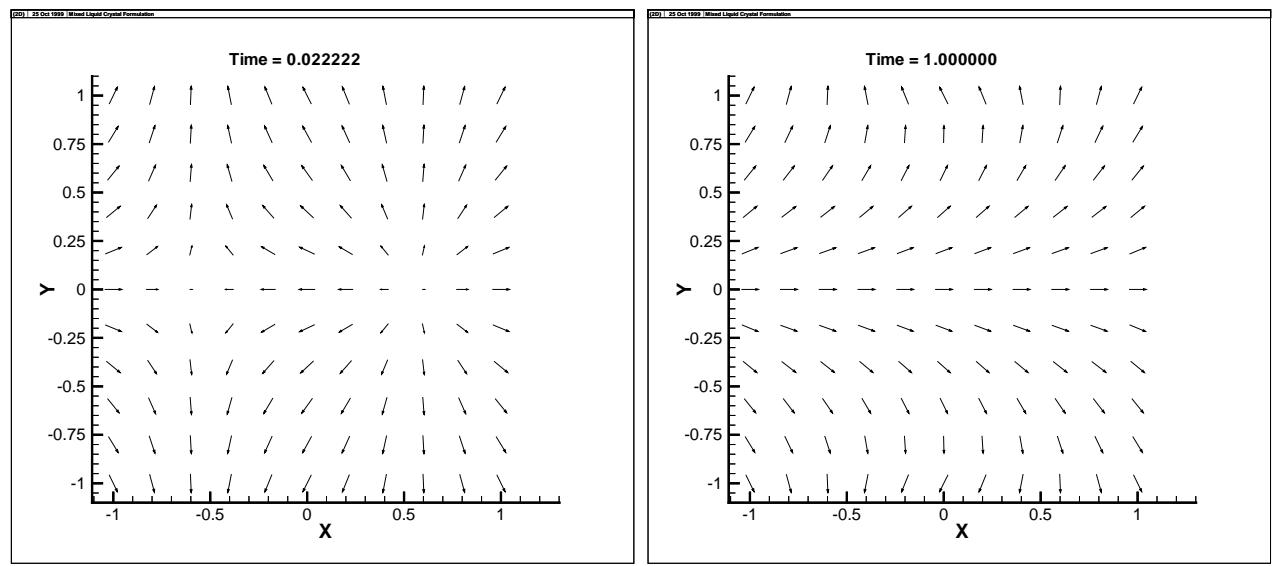

Figure 3. (a) Initial and (b) Final 2d director field
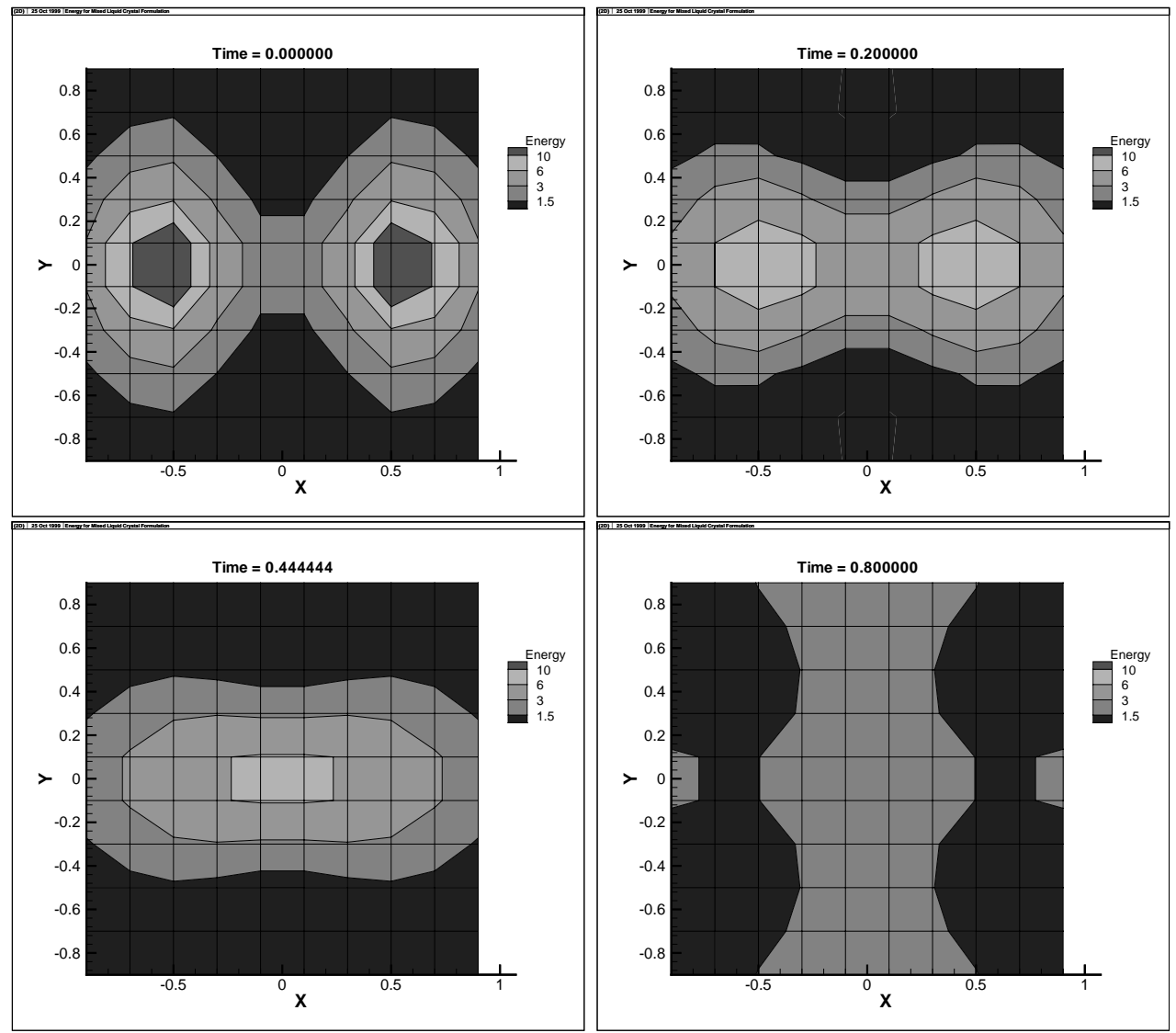

Figure 4. Energy level contours for $2 \mathrm{~d}$ example $(t=0,0.2,0.44,0.8)$. 


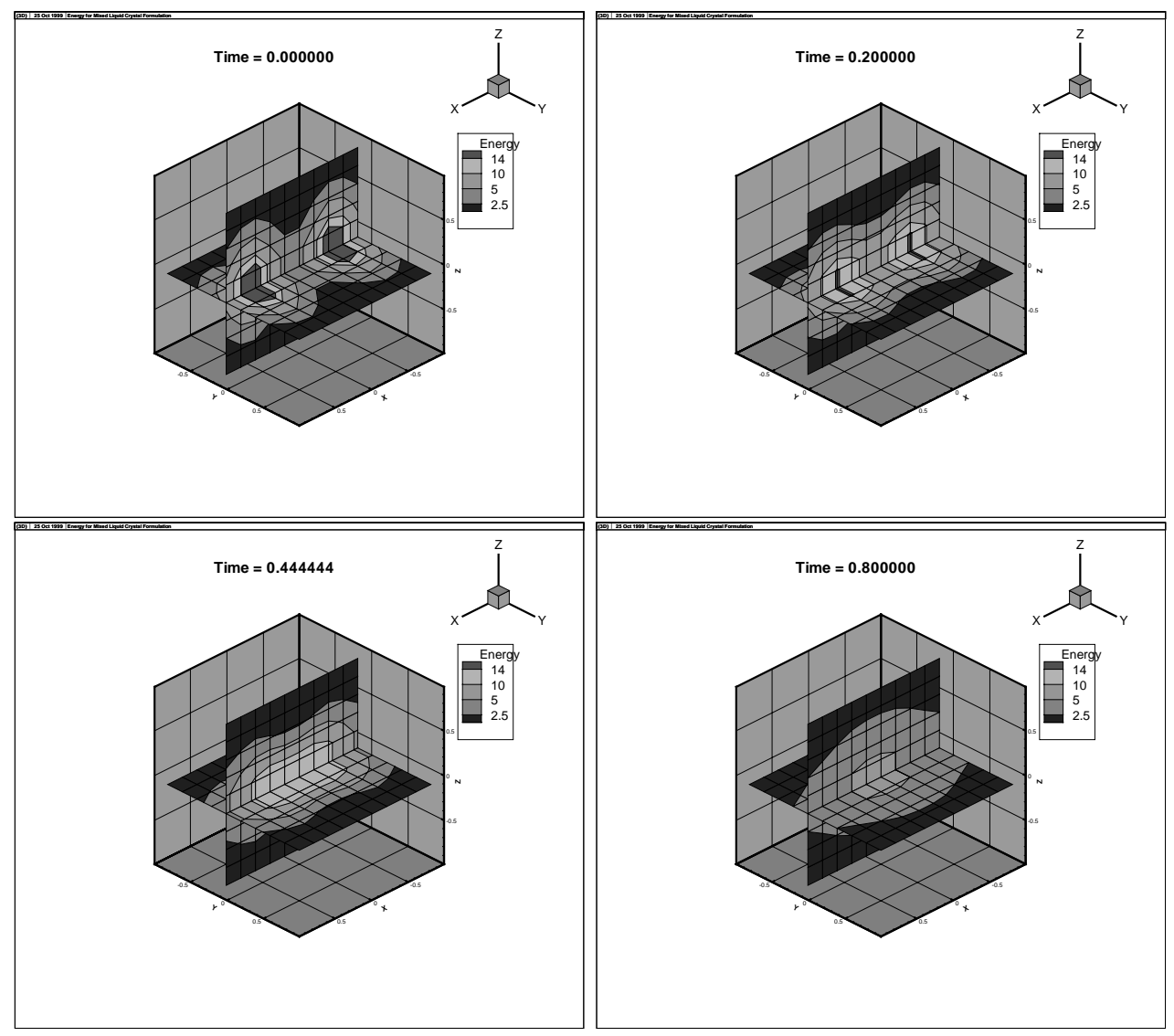

FiguRE 5. Energy level contours for 3d example $(t=0,0.2,0.44,0.8)$.

set to unity, and the penalty parameter $\epsilon^{2}$, and singularity regularization parameter $\varepsilon^{2}$ were both set equal to $0.25^{2}=0.0625$.

Since three dimensional vector plots are difficult to visualize, we chose to plot contours of the energy density

$$
e(t)=(1 / 2)|u|^{2}+(\lambda / 2)|\nabla d|^{2}+\lambda F(d),
$$

to display the three dimensional solution. Recall that equation (1.2) shows that the integral of this quantity, $E(t)$, decreases with time. Figures 4 and 5 show the energy contours for the two and three dimensional problem at several times.

It is clear that the energy is initially concentrated around the singularities, and the region between them quickly takes on a high energy state. After the singularities meet the energy decays towards a low steady state distribution determined by the boundary data. As indicated in Figure 4, for the parameters used here the two dimensional steady state is almost achieved by time $t=0.8$.

In the absence of boundary conditions, energy arguments indicate that in two dimensions singularities of opposite sign will accelerate towards each other; however, in three dimensions these arguments predict constant speeds. Evidence of this appears in the example considered here, since annihilation occurs at a time $t \sim 0.4$ in two dimensions and $t \sim 0.47$ in three dimensions. Figure 6 shows the two dimensional director field close to the 

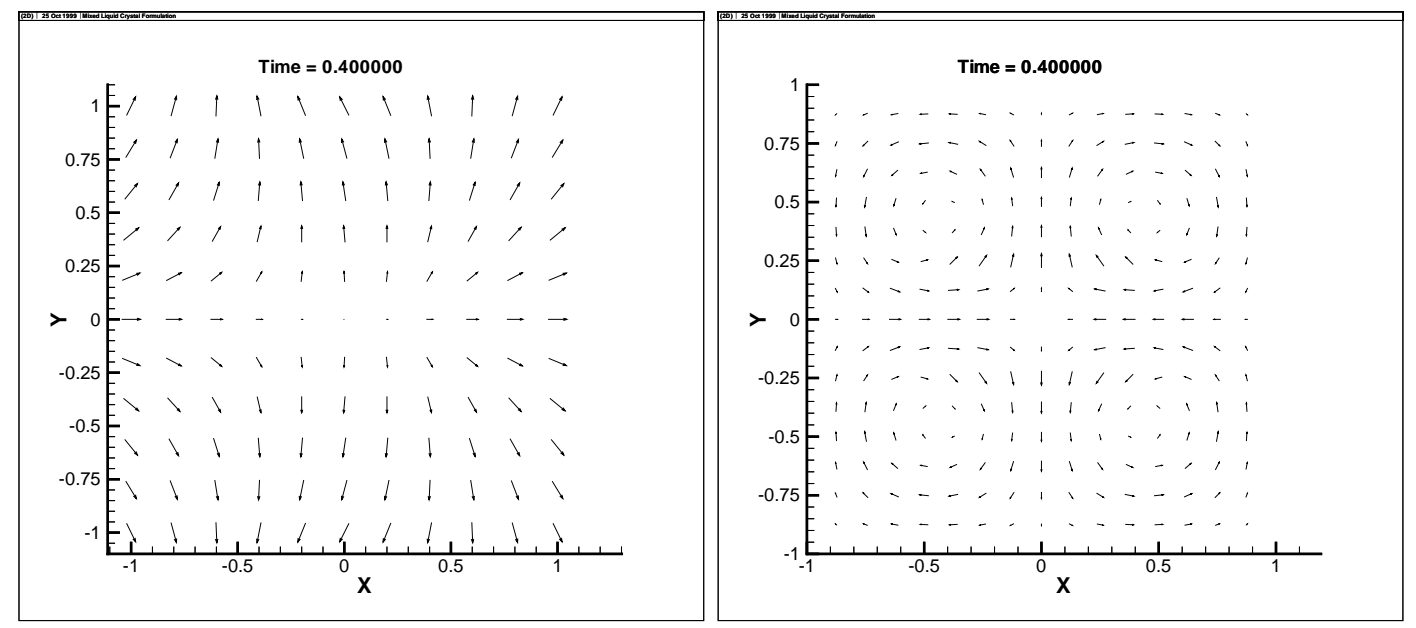

Figure 6. Director and velocity field at annihilation for $2 \mathrm{~d}$ problem.

annihilation time along with the induced velocity field (back flow) ${ }^{3}$. It is interesting to note that the maximum peak velocity occurs at about $t \sim 0.55$ which is well past the annihilation time for the singularities. A section through the three dimensional director field at in the plane $z=0$ at the annihilation looks very similar to the two dimensional configuration shown in Figure 6; however, the vortex structure appears to be truly three dimensional and is harder to visualize. In this situation it is doubtful that the $5 \times 5 \times 5$ grid adequately resolved the velocity field.

\section{REFERENCES}

[1] F. Alouges, A new algorithm for computing liquid crystal stable configurations: The harmonic mapping case. SIAM J. Numer. Anal. 34 (1997) 1708-1726.

[2] F. Alouges and J.M. Ghidaglia, Minimizing Oseen-Frank energy for nematic liquid crystals: algorithms and numerical results. Ann. Inst. H. Poincaré Phys. Théor. 66 (1997) 411-447.

[3] I. Babuška and A.K. Aziz, Survey lecutures on the mathematical foundations of the finite element method, in The mathematical foundations of the finite element method with applications to partial differential equations, A.K. Aziz Ed., New York (1972), Academic Press, 5-359.

[4] F. Bethuel and H. Brezis, Regularity of minimizers of relaxed problems for harmonic maps. J. Funct. Anal. 101 (1991) $145-161$.

[5] F. Bethuel, H. Brezis and F. Helein, Ginzburg-Landau Vorticies. Klumer (1995).

[6] H. Brezis, New developments on the ginzburg-landau model. Topol. Methods Nonlinear Anal. 4 (1994) 227-236.

[7] H. Brezis, J. Coron and E. Lieb, Harmonic maps with defects. Comm. Math. Phys. 107 (1986) 649-705.

[8] F. Brezzi and M. Fortin, Mixed and hybrid finite element methods, no. 15 in Computational Mathematics. Springer-Verlag (1991).

[9] S. Chandrasekhar, Liquid Crystals. Cambridge (1992).

[10] Y.M. Chen and M. Struwe, Regularity for heat flow for harmonic maps. Math. Z. 201 (1989) 83-103.

[11] P.G. Ciarlet, The finite element method for elliptic problems. North-Holland (1978).

[12] R. Cohen, R. Hardt, D. Kinderlehrer, S. Lin and M. Luskin, Minimum energy configurations for liquid crystals: Computational results, in Theory and Applications of Liquid Crystals, J.L. Ericksen and D. Kinderlehrer, Eds., Vol. 5 of The IMA Volumes in Mathematics and its Applicatoins. Springer-Verlag, New York (1987).

[13] R. Cohen, S. Lin and M. Luskin, Relaxation and gradient methods for molecular orientation in liquid crystals. Comp. Phys. 53 (1989) 455-465.

[14] M. Crouzeix and V. Thomee, The stability in $L^{p}$ and $W^{1, p}$ of the $L^{2}$ projection onto finite element function spaces. Math. Comp. 48 (1987) 521-532.

\footnotetext{
${ }^{3}$ We show the velocity field computed with an $8 \times 8$ grid since the simple interpolation used to construct the plots gave poor resolution of the vortex structure on the $5 \times 5$ mesh.
} 
[15] T. Davis and E. Gartland, Finite element analsyis of the Landau-De Gennes minimization problem for liquid crystals. SIAM J. Numer. Anal. 35 (1998) 336-362.

[16] P.G. de Gennes, The Physics Of Liquid Crystals. Oxford (1974).

[17] J. Deang, Q. Du, M. Gunzburger and J. Peterson, Vortices in superconductors: modelling and computer simulations. Philos. Trans. Roy. Soc. London 355 (1997) 1957-1968.

[18] Q. Du and F. Lin, Ginzburg-Landau vortices: dynamics, pinning, and hysteresis. SIAM J. Math. Anal. 28 (1997) $1265-1293$.

[19] Q. Du, R.A. Nicolaides and X. Wu, Analysis and convergence of a covolume approximation of the Ginzburg-Landau model of superconductivity. SIAM J. Numer. Anal. 35 (1997) 1049-1072.

[20] J. Ericksen, Conservation laws for liquid crystals. Trans. Soc. Rheol. 5 (1961) 22-34.

[21] F.C. Frank, On the theory of liquid crystals. Discuss. Faraday Soc. 28 (1958) 19-28.

[22] V. Girault and P.A. Raviart, Finite element approximation of the Navier-Stokes equations, no. 749 in Lecture Notes in Mathematics. Springer Verlag, Berlin, Heidelbert, New York (1979).

[23] M. E. Gurtin, An introduction to continuum mechanics, no. 158 in Mathematics in Science and Engineering. Academic Press (1981).

[24] R. Hardt and D. Kinderlehrer, Mathematical questions of liquid crystal theory, in Theory and Applications of Liquid Crystals, J. L. Ericksen and D. Kinderlehrer Eds., Vol. 5 of The IMA Volumes in Mathematics and its Applicatoins. Springer-Verlag, New York (1987).

[25] R. Hardt, D. Kinderlehrer and F.H. Lin, Existence and partial regularity of static liquid crystal configurations. Comm. Math. Phys. 105 (1986) 547-570.

[26] R. Hardt and F.H. Lin, Stability of singularities of minimizing harmonic maps. J. Differential Geom. 29 (1989) 113-123.

[27] R. Jerard and M. Soner, Dynamics of Ginzburg-Landau vortices. Arch. Rational Mech. Anal. 142 (1998) 99-125.

[28] J. Jost, Harmonic mapping between Riemannian surfaces. Vol. 14 of Proc. of the C.M.A., Australian National University (1983).

[29] F. Leslie, Some constitutive equations for liquid crystals. Archive for Rational Mechanics and Analysis 28 (1968) $265-283$.

[30] F. Leslie, Some topics in equilibrium theory of liquid crystals, in Theory and Applications of Liquid Crystals, J.L. Ericksen and D. Kinderlehrer Eds., Vol. 5 of The IMA Volumes in Mathematics and its Applications. Springer-Verlag, New York (1987) $211-234$.

[31] F.H. Lin, Mathematics theory of liquid crystals, in Applied Mathematics At The Turn Of Century: Lecture notes of the 1993 summer school. Universidat Complutense de Madrid (1995).

[32] F.H. Lin, Some dynamic properties of Ginzburg-Landau vorticies. Comm. Pure Appl. Math. 49 (1996) 323-359.

[33] F.H. Lin and C. Liu, Nonparabolic dissipative systems, modeling the flow of liquid crystals. Comm. Pure Appl. Math. XLVIII (1995) 501-537.

[34] F.H. Lin and C. Liu, Global existence of solutions for the Ericksen Leslie-system. Arch. Rational Mech. Anal. (1998).

[35] S. Lin and M. Luskin, Relaxation methods for liquid crystal problems. SIAM J. Numer. Anal. 26 (1989) 1310-1324.

[36] C. Liu, Dynamic theory for incompressible smectic-A liquid crystals: Existence and regularity. Discrete Contin. Dynam. Systems 6 (2000) 591-608.

[37] C. Liu and N.J. Walkington, Approximation of liquid crystal flows. SIAM J. Numer. Anal. 37 (2000) 725-741.

[38] C.W. Oseen, The theory of liquid crystals. Trans. Faraday Soc. 29 (1933) 883-889.

[39] R. Rannacher and R. Scott, Some optimal error estimates for piecewise linear finite element approximations. Math. Comp. 38 (1982) 437-445

[40] A.H. Schatz and L.B. Wahlbin, On the quasi-optimality in $L_{\infty}$ of the $H_{0}^{1}$ projection into finite element spaces. Math. Comp. 38 (1982) $1-22$.

[41] R. Schoen and K. Uhlenbeck, A regularity theory for harmonic maps. J. Differential Geom. 17 (1982) $307-335$.

[42] J. Shatah, Weak solutions and development of singularities in su(2) $\sigma$-model. CPAM 41 (1988) 459-469.

[43] R. Stenberg, On some three dimensional finite elements for incompressible materials. Comput. Methods Appl. Mech. Engrg. 63 (1987) 261-269.

[44] R. Stenberg, Error analysis of some finite element methods for the Stokes problem. Math. Comp. 54 (1990) $495-508$.

[45] R. Temam, Navier-Stokes Equations. North Holland (1977).

To access this journal online:

www.edpsciences.org 